\title{
Hölder's inequality in Discrete Morrey spaces
}

\author{
Al Azhary Masta ${ }^{1}$, Indra Rukmana ${ }^{2}$, Muhammad Taqiyuddin ${ }^{3}$, and Siti Fatimah ${ }^{4}$ \\ \{alazhari.masta@upi.edu', indra.rukmana09@gmail.com², muhammad.taqiyuddin@uga.edu ${ }^{3}$, \\ Sitifatimah@upi.edu $\left.{ }^{4}\right\}$
}

Department of Mathematics Education, Universitas Pendidikan Indonesia, Jl. Dr. Setiabudi 229, Bandung 40154, INDONESIA ${ }^{1,4}$, Raudhatul Jannah Senior High School, Residence Grand Cilegon, Banten 42426, INDONESIA ${ }^{2}$, Department of Mathematics and Science Education, University of Georgia, Athens, Georgia 30602, United States ${ }^{3}$

\begin{abstract}
Morrey spaces are a generalization of Lebesgue's spaces. There are two categories of Morrey spaces, i.e. continuous Morrey spaces and discrete Morrey spaces. Many authors have discussed about continuous Morrey spaces. In this paper, first we review definitions of these types, and then we present sufficient condition for Hölder's inequality in discrete Morrey spaces and in weak discrete Morrey spaces. One of the keys to prove our results is to use the Hölder's sequence of the balls in $\mathbb{Z}$.
\end{abstract}

Keywords: Hölder's Inequality, Sufficient Condition for Hölder's Inequality, Discrete Morrey Spaces, Weak Discrete Morrey Spaces

\section{Introduction}

Morrey spaces discussions and researches were firstly initiated by C. B. Morrey in [1]. We can consider Morrey spaces as Banach spaces and generalized version of Lebesgue spaces. We can distinguish Morrey spaces into two categories. The first type is 'continuous' Morrey spaces denoted by $\mathcal{M}_{q}^{p}$ and the second one is discrete Morrey spaces which can be denoted by $\ell_{q}^{p}$ for $1 \leq p \leq q<\infty$.

Let us firstly review the definition of 'continuous' Morrey spaces and discrete Morrey spaces. In this article, we define the Morrey space $\mathcal{M}_{q}^{p}\left(\mathbb{R}^{n}\right)$ as the set of all integrable functions $f$ on $\mathbb{R}^{n}$ such that, for $1 \leq p \leq q<\infty$, we have the expression is finite

$$
\|f\|_{\mathcal{M}_{q}^{p}}:=\sup _{a \in \mathbb{R}^{d}, r>0}|B(a, r)|^{\frac{1}{q}-\frac{1}{p}}\left(\int_{B(a, r)}|f(y)|^{p} d y\right)^{\frac{1}{p}} .
$$

By $B(a, r)$, we mean an open ball centered at $a \in \mathbb{R}^{n}$ and equipped with radius $r>0$. Meanwhile, $|B(a, r)|$ represents its Lebesgue measure. Next, observe that $\|\cdot\|_{\mathcal{M}_{q}^{p}}^{p}$ stands for a norm on $\mathcal{M}_{q}^{p}\left(\mathbb{R}^{n}\right)$ and $\mathcal{M}_{q}^{p}\left(\mathbb{R}^{n}\right)$ is Banach spaces equipped with the norm $\|\cdot\|_{\mathcal{M}_{q}^{p}}$. In addition, note that if $q=p$, then we have Morrey space $\mathcal{M}_{q}^{p}\left(\mathbb{R}^{n}\right)$ becomes Lebesgue space $L^{p}\left(\mathbb{R}^{n}\right)$. In the nest paragraph, we will explicate the discrete version with similar fashion.

Now, we will define the discrete Morrey spaces. First, write $S_{m, N}:=\{m-$ $N, \ldots m, \ldots, m+N\}$ in which $m \in \mathbb{Z}$ and $N \in\{0,1,2,3, \ldots\}$. Then, we can define discrete 
Morrey spaces $\ell_{q}^{p}(\mathbb{Z})$ as the set of all sequences $X:=\left(x_{n}\right)_{n \in \mathbb{Z}}$ such that the following condition holds true

$$
\|x\|_{\ell_{q}^{p}(\mathbb{Z})}=\sup _{m \in \mathbb{Z}, N \in \mathbb{N}_{0}}\left|S_{m, N}\right|^{\frac{1}{q}-\frac{1}{p}}\left(\sum_{n \in S_{m, N}}\left|x_{n}\right|^{p}\right)^{\frac{1}{p}}=\sup _{m \in \mathbb{Z}, N \in \mathbb{N}_{0}}\left|S_{m, N}\right|^{\frac{1}{q}-\frac{1}{p}}\|X\|_{\ell_{p}\left(S_{m, N}\right)}<\infty .
$$

Analog with 'continuous' Morrey spaces, if we shift our standpoint by taking $q=p$, then we can consider a Morrey space $\ell_{q}^{p}(\mathbb{Z})=\ell_{p}(\mathbb{Z})$ as a p-summable sequence space. This is indeed not a surprising fact, but we argue that it is worthy to mention here as we will use this important idea later in our main result.

Next, we will define both generalized Morrey spaces and generalized weak Morrey spaces that we will use for the purpose of this article. For $1 \leq p \leq q<\infty$, the weak Morrey space $w \mathcal{M}_{q}^{p}\left(\mathbb{R}^{n}\right)$ is constituted of all measurable functions $f$ on $\mathbb{R}^{n}$ in which the inequality \| $f \|_{w \mathcal{M}_{q}^{p}}<\infty$ is true and its norm is defined by

$$
\|f\|_{w \mathcal{M}_{q}^{p}}:=\sup _{a \in \mathbb{R}^{d}, r, \gamma>0}|B(a, r)|^{\frac{1}{-}-\frac{1}{p}} \gamma|\{x \in B(a, r):|f(x)|>\gamma\}|^{\frac{1}{p}} .
$$

Note that $\|\cdot\|_{w \mathcal{M}_{q}^{p}}$ defines a quasi-norm on $w \mathcal{M}_{q}^{p}\left(\mathbb{R}^{n}\right)$. If $q=p$, then $w \mathcal{M}_{q}^{p}\left(\mathbb{R}^{n}\right)=$ $w L^{p}\left(\mathbb{R}^{n}\right)$. With that being said, we can perceive $w \mathcal{M}_{q}^{p}\left(\mathbb{R}^{n}\right)$ as a way of generalizing the weak Lebesgue space $w L^{p}\left(\mathbb{R}^{n}\right)$.

Again, write $S_{m, N}:=\{m-N, \ldots m, \ldots, m+N\}$ for $m \in \mathbb{Z}$ and $N \in\{0,1,2,3, \ldots\}$. The discrete weak Morrey spaces $\mathrm{w} \ell_{q}^{p}(\mathbb{Z})$ is the set of all sequences $X:=\left(x_{n}\right)_{n \in \mathbb{Z}}$ such that

$$
\|x\|_{\mathrm{w} \ell_{q}^{p}(\mathbb{Z})}=\sup _{m \in \mathbb{Z}, N \in \mathbb{N}_{0, \gamma>0}} \gamma\left|S_{m, N}\right|^{\frac{1}{q}-\frac{1}{p}}\left|\left\{n \in S_{m, N}:\left|x_{n}\right|>\gamma\right\}\right|^{\frac{1}{p}}<\infty .
$$

There are growing and intense discussions on Morrey spaces and its discrete version (see [2-7], etc.). For example, in 2015, Gunawan et al. [9] obtained sufficient and necessary conditions for the inclusion properties of Morrey spaces. Later, in 2019, Gunawan et al. also established the sufficient and necessary conditions for the inclusion properties of discrete Morrey spaces. On the other hand, the following two theorems tells us the sufficient and necessary conditions for generalized Hölder's inequality within 'continuous' Morrey spaces which Ifronika et al. have obtained in 2018.

Theorem 1. Let $1 \leq p \leq q<\infty, 1 \leq p_{1} \leq q_{1}<\infty$, and $1 \leq p_{2} \leq q_{2}<\infty$. Then, these two statements are equivalent:

(1) $\frac{1}{p_{1}}+\frac{1}{p_{2}} \leq \frac{1}{p}$ and $\frac{1}{q_{1}}+\frac{1}{q_{2}}=\frac{1}{q}$.

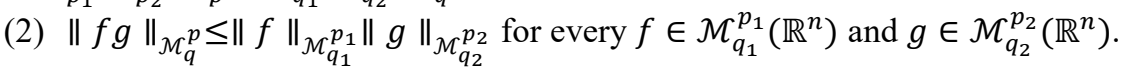

Theorem 2. Let $1 \leq p \leq q<\infty, 1 \leq p_{1} \leq q_{1}<\infty$, and $1 \leq p_{2} \leq q_{2}<\infty$. Then, these two statements are equivalent:

(1) $\frac{1}{p_{1}}+\frac{1}{p_{2}} \leq \frac{1}{p}$ and $\frac{1}{q_{1}}+\frac{1}{q_{2}}=\frac{1}{q}$.

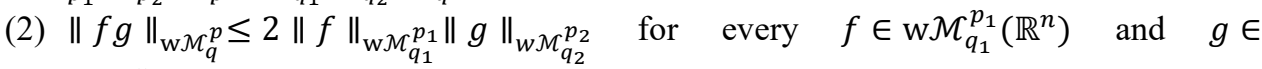
$\mathrm{w}_{\mathcal{M}}^{p_{q_{2}}^{p_{2}}}\left(\mathbb{R}^{n}\right)$. 
In order to continue the discussion and fill in a research gap, we would like to carefully investigate the generalized Hölder's inequality in discrete Morrey spaces and in their weak types. We sought for ways to scrutiny the conditions in which the generalized Hölder's inequality holds true.

To obtain our result, we will use generalized Hölder's inequality in p-summable sequence spaces. We contend that from our results, parameters play significant roles in the Hölder's inequality within the discrete Morrey spaces and their weak type.

\section{Hölder's inequality in discrete Morrey spaces}

Our main findings are summarized in the following theorems. The following theorem describes the sufficient and necessary condition for Hölder's inequality in discrete Morrey spaces and we follow it up with its proof by leveraging the p-summable sequence spaces.

Theorem 3. Let $1 \leq p \leq q<\infty, 1 \leq p_{1} \leq q_{1}<\infty$, and $1 \leq p_{2} \leq q_{2}<\infty$. If $\frac{1}{p_{1}}+\frac{1}{p_{2}} \leq \frac{1}{p}$ and $\frac{1}{q_{1}}+\frac{1}{q_{2}}=\frac{1}{q}$, then we have

$$
\|X Y\|_{\ell_{q}^{p}} \leq\|X\|_{\ell_{q_{1}}^{p_{1}} \| Y} \|_{\ell_{q_{2}}^{p_{2}}}
$$

for every $X:=\left(x_{n}\right) \in \ell_{q_{1}}^{p_{1}}(\mathbb{Z})$ and $:=\left(y_{n}\right) \in \ell_{q_{2}}^{p_{2}}(\mathbb{Z})$.

Proof.

Let $\sum_{i=1}^{m} \frac{1}{p_{i}} \leq \frac{1}{p}$ and $\sum_{i=1}^{m} \frac{1}{q_{i}}=\frac{1}{q}$ hold. Put $\frac{1}{p^{*}}:=\sum_{i=1}^{m} \frac{1}{p_{i}}$. Clearly $p^{*} \geq p$. Now take an arbitrary $S_{m, N} \subseteq \mathbb{N}, X:=\left(x_{n}\right) \in \ell_{q_{1}}^{p_{1}}(\mathbb{Z})$ and $Y:=\left(y_{n}\right) \in \ell_{q_{2}}^{p_{2}}(\mathbb{Z})$. By the Hölder's inequality in $p$-summable sequence spaces [2], we have

$$
\begin{aligned}
\left|S_{m, N}\right|^{\frac{1}{q}-\frac{1}{p}}\|X\|_{\ell_{p}\left(S_{m, N}\right)} & \leq\left|S_{m, N}\right|^{\frac{1}{q} \frac{1}{p^{*}}}\|X\|_{\ell_{p_{*}}\left(S_{m, N}\right)} \\
& \leq\left(\left|S_{m, N}\right|^{\frac{1}{q_{1}}-\frac{1}{p_{1}}}\|X\|_{\ell_{p_{1}}\left(S_{m, N}\right)}\right)\left(\left|S_{m, N}\right|^{\frac{1}{q_{1}}-\frac{1}{p_{1}}}\|X\|_{\ell_{p_{1}}\left(S_{m, N}\right)}\right) .
\end{aligned}
$$

Taking the supremum over we obtain $m \in \mathbb{Z}$ and $N \in \mathbb{N}_{0}$ we have $\|X Y\|_{\ell_{q}^{p}} \leq\|X\|_{\ell_{q_{1}}^{p_{1}} \|}$

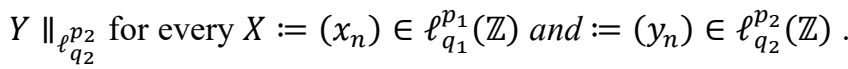

\section{Hölder's inequality in weak discrete Morrey spaces}

Now, we come to suffcient conditions for Hölder's inequalty in Morrey spaces which is presented in the Theorem 4. In the following proof, we use a similar technique which we used in proving Theorem 3 .

Theorem 4. Let $1 \leq p \leq q<\infty, 1 \leq p_{1} \leq q_{1}<\infty$, and $1 \leq p_{2} \leq q_{2}<\infty$. If $\frac{1}{p_{1}}+\frac{1}{p_{2}} \leq \frac{1}{p}$ and $\frac{1}{q_{1}}+\frac{1}{q_{2}}=\frac{1}{q}$, then we have

$$
\|X Y\|_{w \ell_{q}^{p}} \leq 2\|X\|_{\mathrm{w} \ell_{q_{1}}^{p_{1}}\|Y\|_{\mathrm{w} \ell_{q_{2}}^{p_{2}}}}
$$

for every $X:=\left(x_{n}\right) \in \mathrm{w} \ell_{q_{1}}^{p_{1}}(\mathbb{Z})$ and $:=\left(y_{n}\right) \in w \ell_{q_{2}}^{p_{2}}(\mathbb{Z})$. 
Proof.

Let $\sum_{i=1}^{m} \frac{1}{p_{i}} \leq \frac{1}{p}$ and $\sum_{i=1}^{m} \frac{1}{q_{i}}=\frac{1}{q}$ hold. Put $\frac{1}{p^{*}}:=\sum_{i=1}^{m} \frac{1}{p_{i}}$. Clearly $p^{*} \geq p$. Now take an arbitrary $S_{m, N} \subseteq \mathbb{N}, X:=\left(x_{n}\right) \in \mathrm{w} \ell_{q_{1}}^{p_{1}}(\mathbb{Z})$ and $Y:=\left(y_{n}\right) \in \mathrm{w} \ell_{q_{2}}^{p_{2}}(\mathbb{Z})$. By the Hölder's inequality in weak $p$-summbale sequence spaces [2], we have

$$
\begin{aligned}
\left|S_{m, N}\right|^{\frac{1}{q}-\frac{1}{p}}\|X\|_{w \ell_{p}\left(S_{m, N}\right)} & \leq\left|S_{m, N}\right|^{\frac{1}{q}-\frac{1}{p^{*}}}\|X\|_{w \ell_{p *}\left(S_{m, N}\right)} \\
& \leq 2\left(\left|S_{m, N}\right|^{\frac{1}{q_{1}}-\frac{1}{p_{1}}}\|X\|_{w \ell_{p_{1}}\left(S_{m, N}\right)}\right)\left(\left|S_{m, N}\right|^{\frac{1}{q_{1}}-\frac{1}{p_{1}}}\|X\|_{w \ell_{p_{1}}\left(s_{m, N}\right)}\right) .
\end{aligned}
$$

Taking the supremum over we obtain $m \in \mathbb{Z}$ and $N \in \mathbb{N}_{0}$ we have

$$
\|X Y\|_{\mathrm{w} \ell_{q}^{p}} \leq 2\|X\|_{w \ell_{q_{1}}^{p_{1}}}\|Y\|_{w \ell_{q_{2}}^{p_{2}}}
$$

for every $X:=\left(x_{n}\right) \in w \ell_{q_{1}}^{p_{1}}\left(\mathbb{Z}^{n}\right)$ and $:=\left(y_{n}\right) \in \mathrm{w} \ell_{q_{2}}^{p_{2}}\left(\mathbb{Z}^{n}\right)$

\section{Conclusion}

In this short paper, we have shown the sufficient condition for Holder's inequality in discrete Morrey spaces and in their weak type. More specifically, from Theorems 3 and 4, we can state that the condition $\frac{1}{p_{1}}+\frac{1}{p_{2}} \leq \frac{1}{p}$ and $\frac{1}{q_{1}}+\frac{1}{q_{2}}=\frac{1}{q}$ are sufficient conditions for Holder's inequality in discrete Morrey spaces and in their weak type. We would like to call for more mathematical explorations in the related Holder's inequality and Morey spaces research in which we believe, there are still more results to be worthily expanded and deepened.

Acknowledgement. This research was made possible by the following grants supports: Hibah Penguatan Kompetensi UPI and Hibah Afirmasi dan Pembinaan Dosen UPI 2019.

\section{References}

[1] Orlicz, W.: Linear Functional Analysis (Series in Real Analysis Volume 4). World Scientific, Singapore. (1992)

[2] Kufner, A., John, O., and Fucïk, S.: Function Spaces. Noordhoff International Publishing, Czechoslovakia (1977).

[3] Rao, M. M. and Ren Z. D.: Theory of Orlicz spaces. Volume 146 of Monographs and Textbooks in Pure and Applied Mathematics. Marcel Dekker Inc., New York (1991)

[4] Maligranda, L. and Mastylo.: M. Inclusion Mappings between Orlicz Sequence Spaces. Journal of Functional Analysis 176, pp. 264-279 (2000)

[5] Masta, A. A., Gunawan, H., and Budhi, W. S.: Inclusion property of Orlicz and weak Orlicz spaces. J. Math. Fund. Sci. 48-3. pp. 193-203 (2016)

[6] Masta, A. A., Ifronika, and Fatimah, S.: Sufficient and Necessary Conditions for Holder's Inequality in Weighted Orlicz Spaces, to appear in International Journal of Applied Physics and Mathematics 2019

[7] Osançlıol, A.: A Note On The Definition of An Orlicz Space. Afyon Kocatepe University Journal of Science and Engineering, pp. 1-6 (2015) 
[8] Maligranda, L.: Orlicz Spaces and Interpolation. Departamento de Matemática. Universidade Estadual de Campinas. (1989)

[9] Luxemburg, W. A. J.: Banach Function Spaces. Thesis. Technische Hogeschool te Delft (1955) 